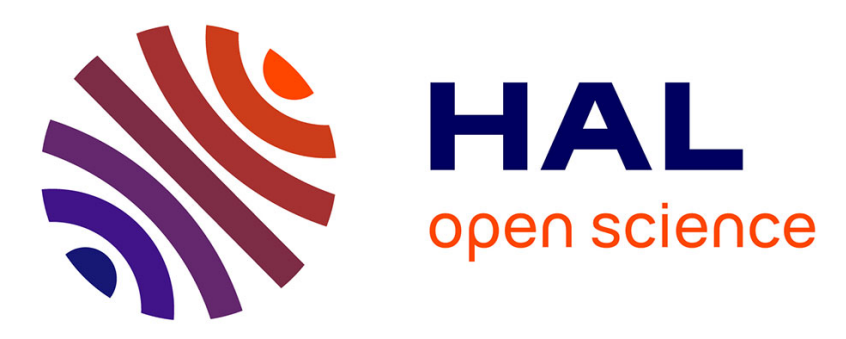

\title{
Battery Aging-Aware Green Cellular Networks with Hybrid Energy Supplies
}

Ali El Amine, Hussein Al Haj Hassan, Loutfi Nuaymi

\section{To cite this version:}

Ali El Amine, Hussein Al Haj Hassan, Loutfi Nuaymi. Battery Aging-Aware Green Cellular Networks with Hybrid Energy Supplies. 2018 IEEE 29th Annual International Symposium on Personal, Indoor and Mobile Radio Communications (PIMRC), Sep 2018, Bologna, Italy. pp.7, 10.1109/PIMRC.2018.8580678 . hal-01985865

\section{HAL Id: hal-01985865 \\ https://imt-atlantique.hal.science/hal-01985865}

Submitted on 18 Jan 2019

HAL is a multi-disciplinary open access archive for the deposit and dissemination of scientific research documents, whether they are published or not. The documents may come from teaching and research institutions in France or abroad, or from public or private research centers.
L'archive ouverte pluridisciplinaire HAL, est destinée au dépôt et à la diffusion de documents scientifiques de niveau recherche, publiés ou non, émanant des établissements d'enseignement et de recherche français ou étrangers, des laboratoires publics ou privés. 


\title{
Battery Aging-Aware Green Cellular Networks with Hybrid Energy Supplies
}

\author{
Ali El-Amine *, Hussein Al Haj Hassan ${ }^{\dagger}$, Loutfi Nuaymi * \\ ${ }^{\star}$ Departement Système Réseaux, Cybersécurité et Droit du numérique (SRCD). IMT-Atlantique. Rennes, France \\ ${ }^{\dagger}$ Department of Computer and Communications Engineering. Faculty of Engineering. American University of \\ Science and Technology. Beirut, Lebanon \\ Email: *firstname.lastname@imt-atlantique.fr, †hhajhassan@ aust.edu.lb
}

\begin{abstract}
Hybrid energy powered cellular networks are key for proposing green and cost-efficient wireless networks. Yet, the related energy management imposes severe challenges to efficiently manage the power allocation between the Renewable Energy (RE) source, the batteries and the smart power grid. The energy storage element (i.e., battery) is prone to irreversible aging mechanisms, requiring intelligent management that takes into account both the energy cost and requirements preventing battery degradation. In this work, we include this important constraint before proposing an energy management and base stations switch off algorithm for a partially RE-equipped cellular network in a variable electricity price environment. Results show that our algorithm outperforms a benchmark algorithm with a gain up to $20 \%$ in terms of electric bill reduction and enhances the battery life time by $35 \%$.
\end{abstract}

\section{INTRODUCTION}

With the explosive increase in Information and Communication Technology (ICT) devices and wireless services, several orders of magnitude base stations (BSs) have been deployed. This has incurred significant energy consumption and carbon footprints. For instance, it is estimated that ICT consumes around $4.7 \%$ of the world's electrical energy, releasing into the atmosphere about $1.7 \%$ of the global $\mathrm{CO}_{2}$ emissions [1]. Moreover, with the evolution of $5 \mathrm{G}$ mobile communications, it is forecasted that the global mobile data traffic will witness an increase of sevenfold between 2016 and 2021 [2]. Under the pressure of this escalation of energy consumption and $\mathrm{CO}_{2}$ emissions, renewable energy (RE) is becoming a promising candidate for green communication systems.

In order to cater for the vision of green $5 \mathrm{G}$ communication systems, a lot of studies have investigated the use of RE in wireless cellular networks as an economic and ecological friendly technique, that can harvest clean and cheap RE from ambient surrounding [3]. Nevertheless, $\mathrm{RE}$ is highly intermittent, thus it requires intelligent management to efficiently use it. In [4], we showed that intelligent usage of RE in a hybrid powered BS system results in higher cost savings in a variable electricity price environment. Based on state of charge $(\mathrm{SoC})$ of the battery and electricity price, we introduced SPAEMA, an energy management algorithm, that decides whether to store or use RE. We then extended the work in [5] to cellular networks with the possibility to switch off BSs, and to adjust the network configuration to increase the utilization of RE. However, both studies ignore the possibility of storing grid energy in the battery for future use. Moreover, [5] considers that all BSs are equipped with RE. Energy cooperation between BSs powered by a hybrid energy source is addressed in [6]. The authors showed that their scheme can significantly improve the grid power consumption of the network. However, their objective focused on the energy consumed by the network rather than its cost. Furthermore, [6] ignores sleep mode technique and considers that all BSs are equipped with RE. Minimizing the grid energy cost in a large-scale green cellular network powered by a hybrid energy source with sleep mode is studied in [7]. However, the authors assumed a RE farm and a centralized energy storage powering all the BSs of the network.

The above mentioned studies use RE as a tool to minimize the grid cost however, they do not take into account the health degradation of the battery that represents a significant cost to the operator. A typical energy storage (i.e., battery) requires expensive investment cost and is prone to irreversible degradations. Some of these degradations are subject to energy exchanges with the battery, and are known as cycle aging. Other degradations occur when the battery is at rest, and they are known as calendar aging [8]. Among the few studies that consider the aging of batteries while studying cellular networks powered by RE is [9]. This study shows that by respecting some battery aging constraints, the battery life span can enhance up to $50 \%$ of its initial state of health per year. This results in an increase in the opex cost savings. However, this work considers the case of a single BS and assumes non-causal information of the RE generation.

In contrast to [9], in this paper we study the case of cellular network where BSs can cooperate under $\mathrm{RE}$ and smart grid (SG) environment, while respecting the constraints imposed on the battery that expand its life span. We consider a large-scale cellular network where a percentage of its sites is equipped with local RE and battery storages. These sites are powered by a hybrid energy source (RE and SG). Under this model, we investigate the allocation of RE, the possibility to store grid energy for future use and the potential to switch off BSs. Furthermore, we propose an online algorithm with causal information about RE generation that minimizes the grid energy bought from the SG. Our simulation results show 
that the proposed solution outperforms the benchmark algorithm by more than $15 \%$ operational cost reduction.

\section{BATTERY MODEL}

Compared with traditional used batteries, i.e., lead acid or nickel cadmium, lithium batteries technologies feature high energy density, high power density, high energy efficiency, large service life and environmental friendliness [10]. However, a typical used battery generates expensive investment cost due to its finite life span. This operational limitation is a result of irreversible degradations from chemical and physical changes, affecting its electrical performance, and hence, degrading its efficiency. In the following, we describe the constraints imposed on the battery that prevent its fast capacity degradation.

A typical battery is described by the following metrics:

1) SoC (State of Charge): It is the ratio of the remaining charge of the battery to the total charge while the battery is fully charged [10].

3) DoD (Depth of Discharge): It is used to describe how much the battery is discharged.

2) SoH (State of Health): It is a figure of merit that represents the condition of the battery compared to its ideal conditions [10]. It can be defined as the ratio of the current capacity and the rated capacity given by the manufacturer:

$$
\operatorname{SoH}(t)=\frac{C_{r e f}(t)}{C_{N}}
$$

The degradation of the battery reference capacity is caused by two aging processes: during operation (i.e. cycling), denoted by cycle aging and at rest, denoted by calendar aging [8]. Even though in this paper we do not focus on calculating the battery capacity degradation, we translate the constraints imposed on the battery in [9] to fit our model, in order to minimize the above mentioned degradations. In the following, we summarize the battery constraints used in our work.

1) Cycle aging: It is the result of the energy exchange with battery. It depends on the battery SoC variations [11]. Fig. 1 illustrates the ideal operating range of SoC that is recommended for lithium batteries [12]. As a result, the lithium battery usage is restricted on a specific range of:

$$
B(t)=[20 \%, 90 \%]
$$

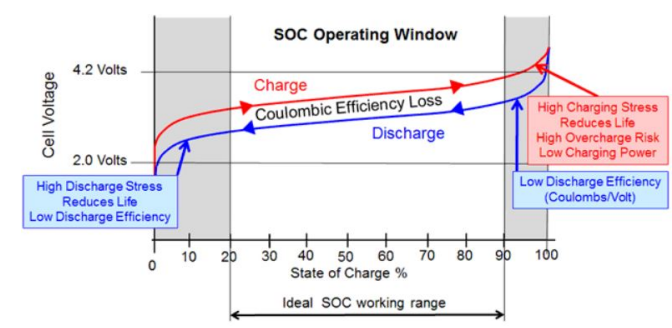

Fig. 1: Recommendation for the operating range of $\mathrm{SoC}$ for lithium ion battery [12]
2) Calendar aging: The battery calendar life is the time elapsed until the battery becomes unusable. It is influenced by the battery temperature and time [9]. Thus, in order to operate the lithium battery in a safe operating area restricted to temperature, high (dis)charge currents should be avoided. Consequently, the following constraint must be respected:

$$
|B(t)-B(t-1)| \leq \alpha \times B(t-1), \forall t=2, \ldots, T .
$$

where $\alpha$ takes a value between 0 and 1 .

In addition to the capacity degradation, the battery witnesses energy losses while (dis)charging. Each time the battery is (dis)charged with $E$ units of energy, only $\eta \cdot E$ is used/stored, where $0 \leq \eta \leq 1$ represents the storage inefficiency.

\section{SySTEM MODEL}

\section{A. Network Description}

We consider a large-scale wireless downlink cellular network, where the traffic loads, the harvested RE, the grid electricity price and the wireless channel gains from each BS to the users are all varying over time in the network. Let $\mathcal{B}=\{1, \ldots, m, \ldots, M\}$ be the set of BSs, and $\mathcal{U}_{m}=\left\{1, \ldots, u, \ldots, k_{m}\right\}$ be the set of users served by BS $m$. Initially, the users are associated with and served by the $\mathrm{BSs}$, based on best signal-to-interference-plus-noise ratio method (Best SINR) that is managed by a centralized energy management unit (EMU). Each user measures the SINRs by using pilot signals from all BSs and sends it to the centralized EMU.

\section{B. System Architecture}

In this work, we assume that every BS receives its energy from the power grid (i.e., the SG). In addition, some BSs are equipped with a RE harvesting source, and a battery to store the harvested energy in order to use it for future transmission. Hence, these BSs are powered by a mix of hybrid energy sources $(\mathrm{SG}+\mathrm{RE})$. We denote by $B S^{\text {grid }}$, the set of BSs powered solely from the grid and not equipped with a RE source, and by $B S^{m i x}$, the set of BSs equipped with a RE source.

A schematic diagram for the system architecture is illustrated in Fig. 2. The amount of RE generated at BS $m \in B S^{m i x}$ during time $t$ is denoted by $R E_{m}(t)$. It can be either stored in the battery $\left(R E_{m}^{b}(t)\right)$ or used directly to power the BS $\left(R E_{m}^{B S}(t)\right)$. Similarly, the SG can be used either to directly power the $\mathrm{BS}\left(p_{m}^{g, B S}(t)\right)$ or to store in the battery $\left(p_{m}^{g, b}(t)\right)$. Consequently, we have the following equality constraints:

$$
\begin{gathered}
R E_{m}(t)=R E_{m}^{B S}(t)+R E_{m}^{b}(t)+w_{m}(t) \\
p_{m}^{g}(t)=p_{m}^{g, b}(t)+p_{m}^{g, B S}(t)
\end{gathered}
$$

where $w_{m}(t)$ is the amount of RE lost at BS $m$.

Due to battery imperfections, a part of the energy is lost while (dis)charging the battery. During (dis)charging, $(1-\eta)$ of energy is lost. The local EMU manages the allocation of RE, whether to be used or stored in the battery. It also decides when and how much to store grid 
energy. On the other hand, the centralized EMU (not shown in the figure) controls the operational mode of the BSs (active or sleep) following our sleep mode strategy presented in [13]: SINR threshold-based method. This algorithm turns-off BSs $\in B S^{\text {grid }}$ according to an SINR Switch-Off Threshold ( SINR $\left._{\mathrm{SOT}}\right)$. A candidate BS $m$ switches off if the SINRs measured by the users served by $m$ from neighboring BSs $m^{\prime}$ are above the SINR $_{\mathrm{SOT}}$ :

$$
\begin{aligned}
& \operatorname{SINR}_{m^{\prime}}(u) \geq \operatorname{SINR}_{\mathrm{SOT}}, \forall u \in U_{m}, m \in B S^{\text {grid }} \\
& \text { and } m^{\prime} \in\{\mathcal{B} \backslash m\} .
\end{aligned}
$$

If Eq. (6) is satisfied, BS $m$ offloads its users to neighboring BSs with the highest received SINRs.

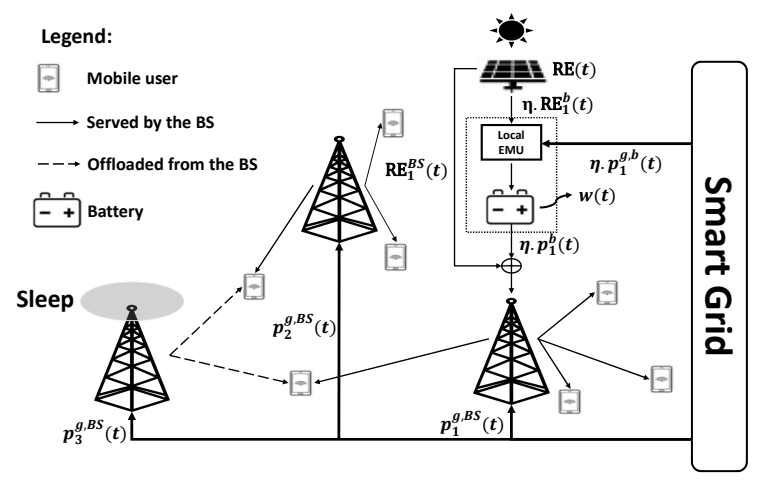

Fig. 2: An example of a cellular network with one BS equipped with a solar panel and a battery.

\section{Energy Consumption Model}

In order to calculate the power consumption of the BS, we use the power model provided by EARTH [14]. Accordingly, the power consumption of a BS consists of a static part $P_{0}$ and a traffic dependent part $P^{\mathrm{Tx}}$. Moreover the power demand depends on the number of active resource blocks (RBs). Because of the nearly linear relationship that exists between the RF power and the BS power consumption, the power consumed by BS $m$ at time $t$ can be expressed as follows:

$$
\begin{aligned}
& p_{m}(t)= \\
& \begin{cases}N_{\mathrm{Tx}}\left(P_{0}+y_{m}(t) \times \Delta_{p} \times P_{m}^{\mathrm{Tx}}(t)\right), & 0<P^{\mathrm{Tx}} \leq P_{\text {max }}, \\
N_{\mathrm{Tx}} p_{m}^{\text {sleep }}(t), & P^{\mathrm{Tx}}=0 .\end{cases}
\end{aligned}
$$

where $N_{\mathrm{Tx}}$ is the number of transceivers. $\Delta_{p}$ and $p_{m}^{\text {sleep }}$ are the load dependent parameter and the power consumption of the BS in sleep mode, respectively. $y_{m}(t)$ is the share of resources used by BS $m$ during time $t$. It is expressed in terms of RBs such as, $y_{m}(t)=\frac{n_{R B}^{t}}{n_{R B}^{\text {Total }}}$, where $n_{R B}^{t}$ and $n_{R B}^{\text {Total }}$ are the number of active RBs at time $t$ and the maximum number of RBs available to the $\mathrm{BS}$, respectively.

\section{Downlink Transmission Model}

We measure the downlink transmission quality between the serving BS $m$ and a user $u$ based on the SINR as follows:

$$
\operatorname{SINR}_{m}(u)=\frac{P_{m}^{\mathrm{Tx}} h_{m}(u)}{\sigma^{2}+\sum_{m^{\prime} \in \mathcal{B}, m^{\prime} \neq m} P_{m^{\prime}} h_{m^{\prime}}(u)}
$$

where $P_{m}^{\mathrm{Tx}}$ is the transmitted power of BS $m, h_{m}(u)$ is the channel gain from BS $m$ to user $u$, which accounts for the path loss and shadowing effect, and $\sigma^{2}$ is the additive white Gaussian noise power density.

We can express the rate offered to user $u$ and served by BS $m$ using Shannon-Hartley theorem as follows:

$$
R_{m}(u)=y_{m}(u) \times \mathrm{BW}_{R B} \times \log _{2}\left(1+\operatorname{SINR}_{m}(u)\right)
$$

where $y_{m}(u)$ is the share of resources allocated to user $u$ by $\mathrm{BS} m$, and $\mathrm{BW}_{R B}$ is the bandwidth of one RB.

\section{E. Energy Storage}

As shown in Fig. 2, the battery can store the harvested energy from the local PV system and the energy purchased from the SG. To make sure that the BS $m$ uses only the energy that is available before the beginning of each time slot, we need the following energy causality constraints:

$$
\begin{aligned}
& B_{m}(t)=B_{0} \\
& +\sum_{i=1}^{t-1}\left(\eta\left(R E_{m}^{b}(i)+p_{m}^{g, b}(i)\right)-p_{m}^{b}(i)-w_{m}(i)\right) \geq 0, \\
& \forall t=1, \ldots, T, \forall m \in B S^{m i x} .
\end{aligned}
$$

The term $w_{m}(t)$ introduced refers to the amount of RE lost at the end of time $t$. This is due to the limited battery capacity, $B_{\max }$. Consequently, the battery has to discard the excess harvested energy, $w_{m}$, to satisfy the following battery capacity constraints:

$$
B_{m} \leq B_{\max }, \forall t=1, \ldots, T, \forall m \in B S^{m i x} .
$$

\section{PROBLEM ForMUlation}

Denote the real-time price of electricity at each time step $t$ as $a(t)$. We aim at minimizing the grid energy bought from the SG while satisfying the users' quality of service (i.e., minimum rate requirement), and reducing the battery aging. This is achieved by allocating the power flow between the RE source, the battery storages, the SG and the BSs. In addition, we consider switching-off some BSs, and resource allocation between active BSs and their served users to save energy. In the following, we consider that $\left(\mathbf{p}, \mathbf{p}^{\mathbf{g}, \mathbf{B S}}, \mathbf{p}^{\mathbf{g}, \mathbf{b}}, \mathbf{p}^{\mathbf{b}}, \mathbf{w}, \mathbf{R E}^{\mathbf{B S}}, \mathbf{R E}^{\mathbf{b}}\right)=$ $\left(\phi_{t, m}\right)_{t=1, \ldots, T, m \in B S^{m i x}} \in \mathbb{R}_{+}^{T \times B S^{m i x}}$ where $\boldsymbol{\phi}$ is a matrix variable, $\boldsymbol{\pi}=\left(\boldsymbol{\pi}_{t, m}\right)_{t=1, \ldots, T, m \in \mathcal{B}} \in\{0,1\}$, $\mathbf{x}=\left(\mathbf{x}_{t, m, u}\right)_{t=1, \ldots, T, m \in \mathcal{B}, u, \in \mathcal{U}_{\Uparrow}} \in\{0,1\}$ and

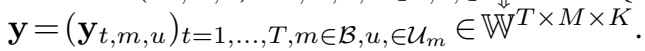

$$
\begin{aligned}
& \min _{\substack{\mathbf{p}, \mathbf{p}^{\mathbf{g}, \mathbf{B S}}, \mathbf{p}^{\mathbf{g}, \mathbf{b}}, \mathbf{p}^{\mathbf{b}}, \mathbf{x}, \mathbf{y}, \boldsymbol{\pi}, \boldsymbol{w}, \mathbf{R E}^{B S}, \mathbf{R E}^{b}}} \sum_{t=1}^{T} \operatorname{Cost}(t)=\sum_{t=1}^{T} \sum_{m \in \mathcal{B}} a(t)\left(\pi _ { m } ( t ) \left(p_{m}(t)+\right.\right. \\
& \left.\left.p_{m}^{g, b}(t)-\eta \cdot p_{m}^{b}(t)-R E_{m}^{B S}(t)\right)+\left(1-\pi_{m}(t)\right) p_{m}^{\text {sleep }}(t)\right)
\end{aligned}
$$


Given that:

$$
p_{m}^{g, B S}(t)=p_{m}(t)-\eta \cdot p_{m}^{b}(t)-R E_{m}^{B S}(t)
$$

and from Eq. (5), we can rewrite (12) as follows:

$$
\begin{gathered}
\min _{\substack{\mathbf{p}, \mathbf{p}^{\mathbf{g}, \mathbf{B S}}, \mathbf{p}^{\mathbf{g}}, \mathbf{b} \\
\mathbf{p} \mathbf{b}, \mathbf{x}, \mathbf{y}, \boldsymbol{\pi}, \boldsymbol{w}, \mathbf{R E}^{\mathbf{R}}, \mathbf{R E}^{B S}, \mathbf{R E}^{b}}} \sum_{t=1}^{T} \sum_{m \in \mathcal{B}} a(t)\left(\pi_{m}(t) p_{m}^{g}(t)+\right. \\
\left.\left(1-\pi_{m}(t)\right) p_{m}^{\text {sleep }}(t)\right)
\end{gathered}
$$

subject to:

$$
\begin{aligned}
& y_{m}(t, u) \times \mathrm{BW}_{R B} \times \log _{2}(1+\operatorname{SINR}(t, u)) \geq R^{r e q} \\
& \forall u \in \mathcal{U}_{m}, \forall m \in \mathcal{B}, \forall t=1 \ldots T \\
& p(t)=p^{g, B S}(t)+\eta \cdot p^{b}(t)+R E^{B S}(t) \\
& \forall t=1 \ldots T \\
& \sum_{u \in \mathcal{U}} y_{m}(u) \leq 1, \forall m \in \mathcal{B} \\
& \sum_{m \in \mathcal{B}} x_{u}(m)=1, x_{u}(m) \in\{0,1\}, \forall u \in \mathcal{U} \\
& \mathbf{p} \geq 0, \mathbf{p}^{\mathbf{g}} \geq 0, \mathbf{p}^{\mathbf{b}} \geq 0, \mathbf{p}^{\mathbf{g}, \mathbf{B S}} \geq 0, \mathbf{p}^{\mathbf{g}, \mathbf{b}} \geq 0 \\
& \mathbf{w} \geq 0, \mathbf{R E}^{B S} \geq 0, \mathbf{R E}^{b} \geq 0
\end{aligned}
$$

Other constraints: (2),(3),(4),(5),(10),(11).

where $\mathbf{x}$ represents the BS-user association matrix, and $\pi_{m}$ is a binary variable that represents the state mode of BS $m\left(\pi_{m}=1\right.$ : $\mathrm{BS}$ is active; $\pi_{m}=0$ : $\mathrm{BS}$ is in sleep mode). The first set of constraints (15) guarantee a minimum rate requirement for the users in order to satisfy a certain QoS level. Next, we have the RE usage, grid power usage and BS power equality constraints in (4),(5) and (16), respectively. The causal energy constraint in (10) ensures that the total RE used up until time $t$ will not exceed the available amount that was harvested and stored in the previous time slot $t-1$. On the other hand, (11) is the battery capacity constraint. Consequently, in case of battery overflow, the excess energy must be discharged through the auxiliary variable $w$. (2) and (3) restrict the SoC operating range and the (dis)charging current of the battery, respectively. Finally, the resource allocation and BS-User association constraints are given in (17) and (18). The last set of constraints in (19) depict the non-negative constraints of the vectors.

The problem formulated above is a mixed-integer non-linear programming (MINLP) problem, which is hard to solve due to its non-convexity. In the next section, we propose a new online sub-optimal solution. And we will show that it performs better than some existing online algorithms.

\section{DESCRIPTION OF THE PROPOSED ALGORITHM}

Due to the complexity and the non-causality of RE information in the problem formulated above, in this section we propose a distributed online solution that manages the energy allocation between the RE source, the SG and the batteries of the BSs equipped with RE. Hence, in the following, we develop an algorithm that doesn't require information about future RE generation.

\section{A. Grid Energy Purchase Policy and Resource sharing}

According to Eq. (7), the minimum amount of power required to power the BS up until the observation time $T$ is:

$$
\begin{aligned}
P^{\text {min }}(t) & =\sum_{i=t}^{T} p^{\text {min }}(i) \\
& =\sum_{i=t}^{T}\left(P_{0}+\frac{1}{n_{R B}^{\text {Total }}} \times \Delta_{p} \times P^{\mathrm{Tx}}(i)\right)
\end{aligned}
$$

Eq. (20) gives the minimum power required for the BS to be in operational mode, i.e., by serving only one $\mathrm{RB}$, from time $t$ until the end of the day.

Proposition 6.1 (Grid energy purchase policy): The amount of purchased energy from the grid should satisfy the following conditions:

1) Condition \#1: The current grid electricity price is low enough, $a(t) \leq \min (a(t+1) \ldots a(T))$.

2) Condition \#2: The future harvested energy is low, $\sum_{i=t}^{T} R E(i) \leq P^{\min }(t)$.

If the above conditions are satisfied:

$$
\begin{aligned}
& p^{g, b}(t)= \\
& \min \left(\alpha \cdot\left(B_{\max }-B(t)\right), \alpha \cdot\left(P^{\min }(t)-\sum_{i=t}^{T} R E(i)\right)\right)
\end{aligned}
$$

Proposition 6.1 decides when to purchase grid energy, and how much to store in the battery. Condition \# 1 allows buying grid energy only when the price is at its lowest compared to the future price. This policy is in accord with the daily grid energy price that is low at the beginning of the day and assumes future knowledge of the electricity price [15]. However, condition \# 2 requires the exact amount of RE that will be harvested in the future. Since this information is missing, we will predict this energy using weather forecast programs, such as [16]. Thus, we can rewrite condition \# 2 as follows:

$$
G(t) \leq P^{\min }(t)
$$

where $G(t)$ is the green energy budget predicted from time $t$ until the end of the observation time, $T$.

Proposition 6.2 (Max-Min Fairness Algorithm): In order to share the resources of the BS among the users it is serving in order to satisfy their QoS requirements, we use the Max-Min fairness algorithm detailed in [17], [13] The algorithm starts by computing the share of resources needed for each user. Then, it will divide the resources evenly among them. If a user gets more resources than his requirement, additional resources will be divided among other users that haven't yet achieved their requirements. If all users are satisfied, excess resources will be turned off 
to save energy. In our model, we consider that the BS has a fixed number of resources expressed in terms of RBs.

\section{B. Joint Power Allocation and Resource Sharing with Sleep Mode Algorithm (JPARS-SM)}

The general algorithm procedure, summarized in Algorithm 1, is detailed in the following. We start by setting the SINR threshold (SINR SOT $_{\text {) }}$ that specifies when the BS can switch to sleep mode. This threshold is set in order to obtain the best trade-off between QoS and energy savings [13]. Then after setting the BS-Users association and resource allocation matrices ( $\mathrm{x}$ and $\mathrm{y}$ ), JPARS-SM scans for the BSs solely powered by the power grid and checks the possibility to switch them to sleep mode, one by one, starting with the BS having the lowest load following Eq. (6). Finally, we apply the battery aging and price aware algorithm (i.e., BAPA) on the remaining BSs (equipped with RE) to efficiently allocate the power between the RE source, the SG and the battery in order to minimize the electric bill of the operator. In the next subsection, we detail BAPA algorithm.

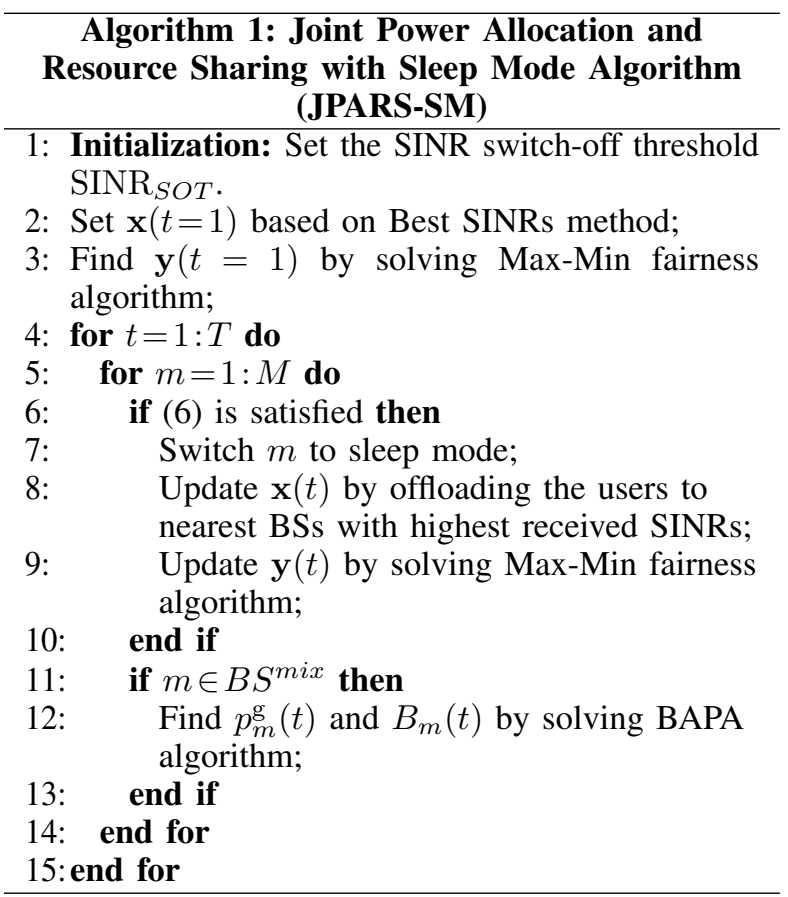

\section{Battery Aging and Price-Aware (BAPA) Algorithm}

BAPA algorithm is divided into three parts. The first part (Lines 2-6) finds how much grid energy to store in the battery, and when to purchase it following Section V-A. The second part (Line 7) finds the allowed amount of RE that can be stored in the battery $\left(R E_{m}^{b}\right)$, while respecting the battery constraints in (2) and (3). Finally, the last part (Lines 8-14) decides whether to use the battery to power the BS or save its energy for future use (i.e., when there is enough RE).

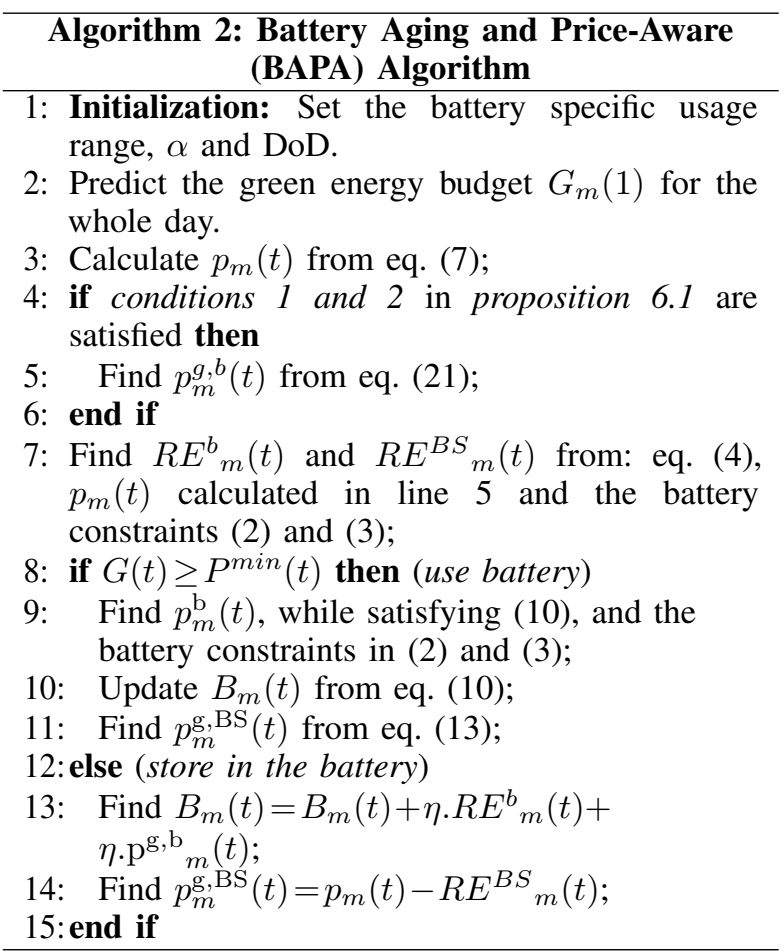

\section{Simulation AND Results}

In this section, we provide simulation results to evaluate the behavior of the system under the battery model proposed in Section II. First, we compare the operational cost gains between imposing the battery aging constraints and ignoring them. As a benchmark, we use the algorithm provided in [4]: SPAEMA. Then we evaluate the performance of our proposed solution and compare it with the benchmark algorithm.

We obtain our results via Monte-Carlo method using MATLAB. Furthermore, we consider the month of June as an example to harvest RE, due to its high solar potential. We set our observation time $T=24$ hours and divide each hour into 10 steps to have a better resolution. During each time step, the amount of RE harvested is assumed to be constant, as well as the number of served users. Only the channel gains and the distribution of the users change during this short period. We summarize the simulation parameters in Table I.

\section{A. System Evaluation under Battery Constraints}

The battery aging constraints limit its usage. Thus, on the short term, we expect to observe a degradation in the system performance, in terms of grid energy electric bill. In Fig. 3, we compare the grid cost over one day for different percentage of sites equipped with RE. We observe a decrease of $5 \%$ in the cost reduction gain with strict battery utilization compared with aggressive utilization that ignores battery degradations. The $23 \%$ price reduction obtained when none of the BSs are equipped with RE source is a result of the resource allocation (Max-Min fairness) algorithm that turns off excess RBs to save energy. However, and by looking at the long term 
TABLE I: Parameters' values and assumptions

\begin{tabular}{|c|c|}
\hline Parameters & Values \\
\hline \hline Number of BSs & 25 \\
\hline Number of sectors & 3 \\
\hline Bandwidth & $10 \mathrm{MHz}, \mathrm{FDD}$ \\
\hline Maximum transmitted power & $43 \mathrm{dBm}$ \\
\hline Inter-cell distance & $1000 \mathrm{~m}$ \\
\hline RB & 50 \\
\hline BW $_{R B}$ & $180 \mathrm{KHz}$ \\
\hline Max. number of users K & 400 \\
\hline User min. required rate & $1 \mathrm{Mb} / \mathrm{s}$ \\
\hline Noise power & $-174 \mathrm{dBm} / \mathrm{Hz}$ \\
\hline$P_{0}$ & $118.7 \mathrm{~W}$ \\
\hline$\Delta_{p}$ & 5.32 \\
\hline Number of slots per hour period & 10 \\
\hline Current restriction $\alpha$ & 0.3 \\
\hline Depth of Discharge DoD & $70 \%$ \\
\hline Battery energy efficiency $\eta$ & 0.96 \\
\hline SINR & $-32 \mathrm{~dB}$ \\
\hline
\end{tabular}

operational cost of the system in Fig. 4, we observe that while respecting the battery aging constraints, we lose on average $40 \%$ of the battery initial capacity after one year compared to $75 \%$ when these constraints are ignored The latter results in a decrease in the operational cost of about $4 \%$ compared to BAPA algorithm, after one year The details of estimating the battery capacity degradations are derived in [9] and are omitted here for brevity. Even though these degradations are pessimistic, they serve as a benchmark to compare the different algorithms.

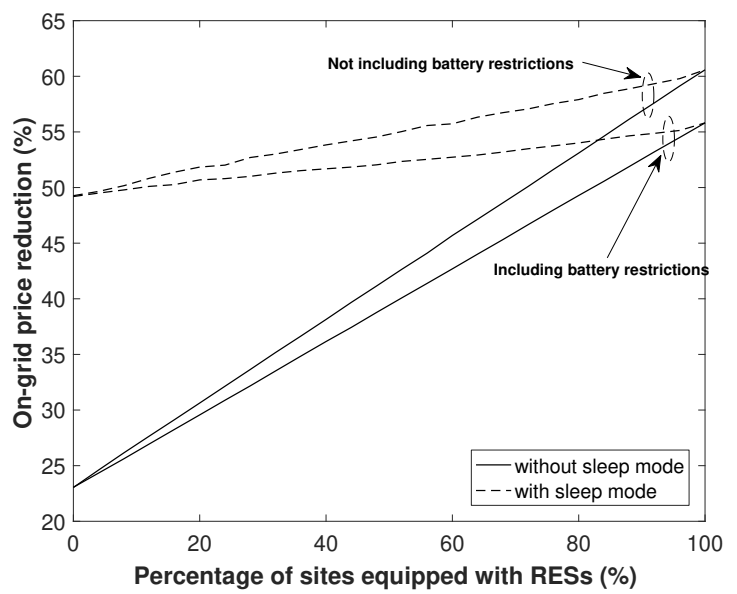

Fig. 3: System performance over one day under battery aging constraints. The solar panels harvest $50 \%$ of the total BS energy consumption and equipped with a $3 \mathrm{KWh}$ batteries.

\section{B. Performance of BAPA Algorithm}

In this section, we evaluate the performance of BAPA by comparing it with the benchmark scheme. We evaluate this benchmark under two cases: with and without battery degradation constraints. BAPA algorithm outperforms the benchmark for the distinct cases and for the different percentage of sites equipped with RE, as shown in Fig.

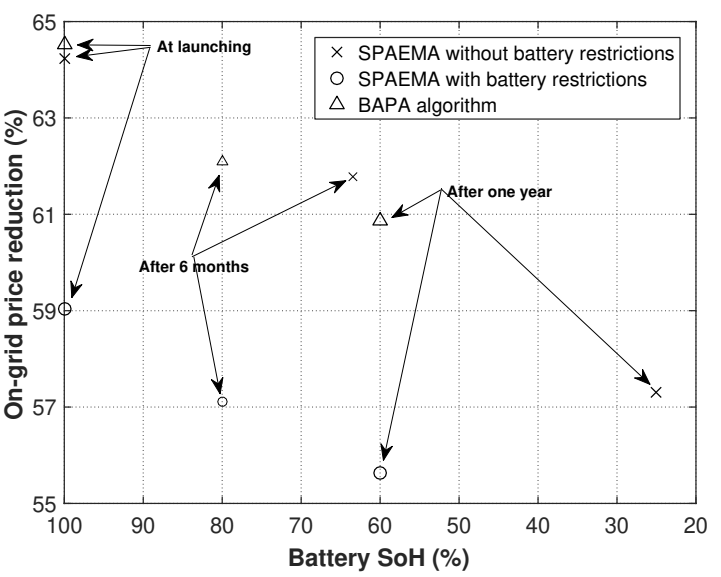

Fig. 4: Grid energy cost savings with the average evolution of the battery SoH after one year.

5. With high daily average RE, our algorithm surpasses SPAEMA by more than $15 \%$ cost reduction points. This amelioration is the result of storing grid energy when the price is low, and then use it at high electricity prices. Such operation avoids using grid energy at high prices and thus, decreases the operational cost of the operator. The down performance in the case of low average RE, is due to the limited battery capacity that restricts the proper energy allocation between the energy stored from the grid and from the RE source. With a small battery capacity, our algorithm stores energy from the grid but is not able to use it efficiently due to the battery constraint in Eq. (3) that limits the (dis)charging capacity during a time step.

We illustrate the waste of energy in Fig. 6 and 7. The waste of energy reduces when applying sleep mode, as shown in Fig. 6. When some BSs switch to sleep mode, some of their users offload to BSs equipped with RE, increasing their energy consumption and thus, their RE usage. This will avoid battery overflow. From Fig. 7, BAPA algorithm shows slightly higher energy losses in the case of large battery capacity. This is because the (dis)charging rate limit of the battery increases with its size allowing more energy to be stored/used. And, since BAPA algorithm allows the possibility to store energy from the grid for future use, more energy is lost (due to battery inefficiency) compared to the benchmark algorithm that ignores storing energy from the grid.

\section{CONCLUSION}

In this paper, we have addressed the grid energy cost of a hybrid energy powered cellular network by jointly considering RE management, resource allocation and sleep mode, under a battery aging constraint model. First, we have compared the cost gain achieved between strict battery utilization that expands its life span, and aggressive utilization that ignores battery degradations. Simulation results have shown that even though the operator loses $5 \%$ points in operational cost savings when restricting the battery usage on the short term, the battery calendar aging will be enhanced by $35 \%$ points. Then, we have proposed a new online algorithm that manages the energy allocation between the RE source, the 


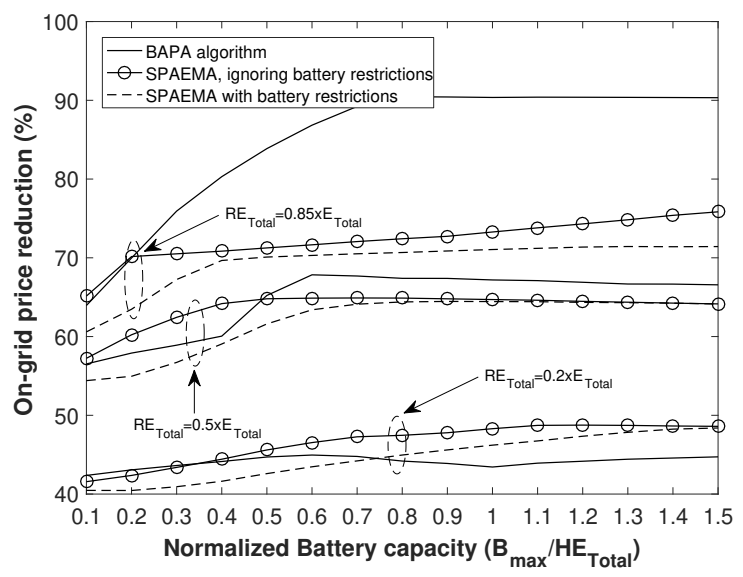

Fig. 5: Performance evaluation comparison of our algorithm and the benchmark algorithms for different average amount of $\mathrm{RE}$ (all sites are equipped with RE).

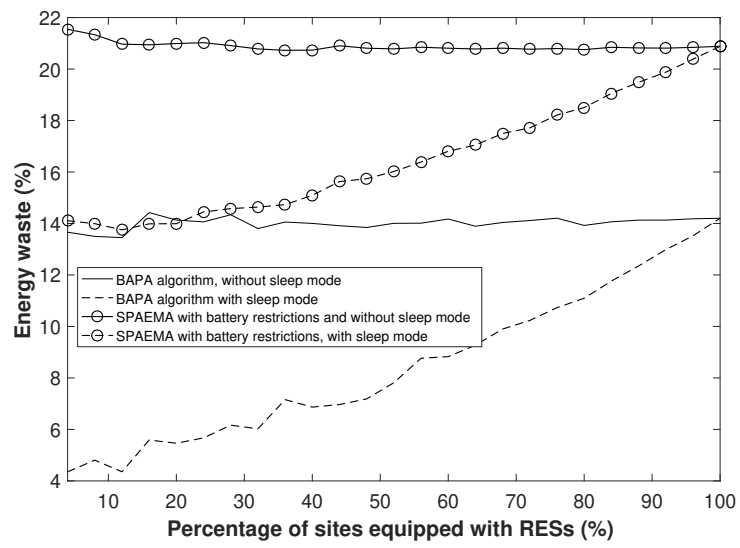

Fig. 6: The waste in energy caused by battery overflow and battery inefficiency with and without sleep mode.

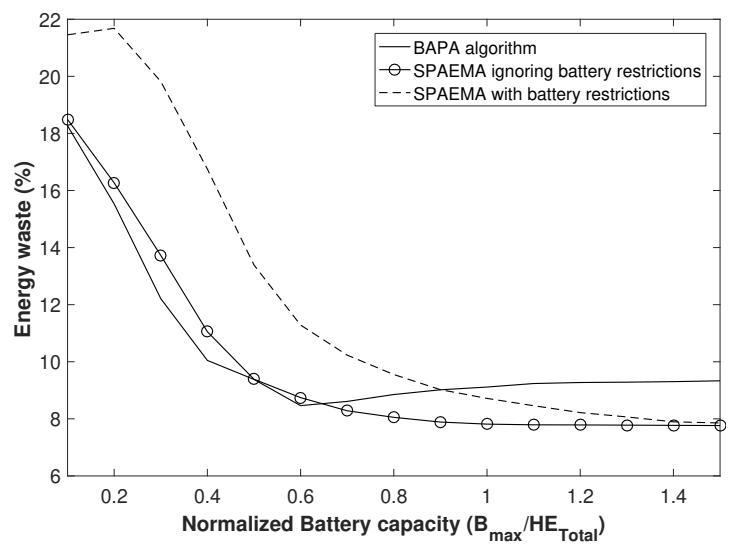

Fig. 7: Comparison of the RE waste for the different algorithms.

smart grid and the battery, with the possibility to store grid energy for future use, taking into account the battery aging constraint model. Our results outperforms the benchmark algorithm by around $20 \%$ more cost reduction points, and surpasses the case where the battery is aggressively used by $15 \%$ points. In future work, we intend to estimate the evolution of the total cost of management over time. Despite the decrease in cost reduction in the short term, longer battery life would reduce the investment cost and would decrease the total cost. The study of the time evolution of the system performance based on the battery model should provide interesting recommendations for green cellular networks.

\section{ACKNOWLEDGMENTS}

The authors would like to acknowledge the support of the French Ministry of Industry for supporting this work under SooGREEN project.

\section{REFERENCES}

[1] E. Gelenbe and Y. Caseau, "The impact of information technology on energy consumption and carbon emissions," 2015.

[2] Cisco Visual Networking Index: Global Mobile Data Traffic Forecast Update, 2016-2021. Cisco, Tech. Rep., February 2017.

[3] H. A. H. Hassan, L. Nuaymi, and A. Pelov, "Renewable energy in cellular networks: A survey," in IEEE Online Conference on Green Communications (OnlineGreenComm), pp. 1-7, Oct 2013.

[4] H. A. H. Hassan, L. Nuaymi, and A. Pelov, "Classification of renewable energy scenarios and objectives for cellular networks," in IEEE 24th Annual International Symposium on Personal, Indoor, and Mobile Radio Communications (PIMRC), pp. 2967-2972, Sept 2013.

[5] H. A. H. Hassan, A. Pelov, and L. Nuaymi, "Cost-efficient radio resource allocation in hybrid energy cellular networks," in 2014 IEEE Global Communications Conference, pp. 2472-2478, Dec 2014.

[6] R. Ramamonjison and V. K. Bhargava, "Energy allocation and cooperation for energy-efficient wireless two-tier networks," IEEE Transactions on Wireless Communications, vol. 15, pp. 6434-6448, Sept 2016.

[7] Y. Che, L. Duan, and R. Zhang, "Dynamic base station operation in large-scale green cellular networks," IEEE Journal on Selected Areas in Communications, vol. PP, no. 99, pp. 1-1, 2016

[8] M. Broussely, P. Biensan, F. Bonhomme, P. Blanchard, S. Herreyre, K. Nechev, and R. Staniewicz, "Main aging mechanisms in li ion batteries," Journal of Power Sources, vol. 146, no. 1, pp. 90 $-96,2005$. Selected papers pressented at the 12th International Meeting on Lithium Batteries.

[9] M. Mendil, A. De Domenico, V. Heiries, R. Caire, and N. Hadjsaid, "Battery aging-aware energy management of green small cells powered by the smart grid," EURASIP Journal on Wireless Communications and Networking, p. 127, Jul 2017.

[10] L. Lu, X. Han, J. Li, J. Hua, and M. Ouyang, "A review on the key issues for lithium-ion battery management in electric vehicles," Journal of Power Sources, vol. 226, pp. 272 - 288, 2013.

[11] Y. Riffonneau, S. Bacha, F. Barruel, and S. Ploix, "Optimal power flow management for grid connected pv systems with batteries," IEEE Transactions on Sustainable Energy, vol. 2, pp. 309-320, July 2011.

[12] "Lithium battery failures." http://mpoweruk.com/lithium. last checked: February 2018.

[13] A. E. Amine, H. A. H. Hassan, and L. Nuaymi, "Analysis of energy and cost savings in hybrid base stations power configurations," in IEEE Vehicular Technology Conference (VTC Spring), 2018.

[14] G. Auer, V. Giannini, C. Desset, I. Godor, P. Skillermark, M. Olsson, M. A. Imran, D. Sabella, M. J. Gonzalez, O. Blume, and A. Fehske, "How much energy is needed to run a wireless network?," IEEE Wireless Communications, vol. 18, pp. 40-49, October 2011.

[15] "Epex, european power exchange spot information." last checked: February 2018

[16] "PVWatts calculator." last checked: February 2018.

[17] M. Ghorbanzadeh, A. Abdelhadi, and C. Clancy, Utility Functions and Radio Resource Allocation, pp. 21-36. Cham: Springer International Publishing, 2017. 